\title{
The effect of insemination methods on in vitro maturation outcomes
}

\author{
Pallop Pongsuthirak \\ Department of Obstetrics and Gynecology, Buddhachinaraj Hospital Medical School, Phitsanulok, Thailand
}

\begin{abstract}
Objective: The aim of this study was to compare the effects of conventional insemination (in vitro fertilization [IVF]) and intracytoplasmic sperm injection (ICSI) on the fertilization, developmental competence, implantation potential, and clinical pregnancy rate of embryos derived from in vitro matured oocytes of patients with polycystic ovary syndrome (PCOS).

Methods: A prospective study was carried out among 38 PCOS patients who had undergone In vitro maturation (IVM) treatment. In total, 828 immature oocytes were collected from 42 cycles and randomly assigned for insemination by IVF ( 416 oocytes) or ICSI (412 oocytes). After fertilization, the embryos were cultured until the blastocyst stage and single embryos were transferred after endometrial preparation and under ultrasound guidance.

Results: No significant differences were found in the maturation rate ( $78.1 \%$ vs. $72.6 \%$ for IVF and ICSI insemination, respectively; $p=0.076)$, fertilization rate $(59.4 \%$ vs. $66.9 \%$ for IVF and ICSI insemination, respectively; $p=0.063)$, or the formation of good-quality blastocysts ( $40.9 \%$ vs. $46.5 \%$ for IVF and ICSI insemination, respectively; $p=0.314$ ). Implantation and clinical pregnancy also did not show significant differences.

Conclusion: There was a comparable yield of in vitro matured oocytes derived from PCOS patients in terms of fertilization, blastocyst formation, implantation rate, and clinical pregnancy between IVF and ICSI insemination. These findings provide valuable insights for choosing assisted reproductive treatment in women with PCOS, as IVM offers promising outcomes and is less invasive and less costly.
\end{abstract}

Keywords: In vitro fertilization; In vitro maturation; Insemination; Intracytoplasmic sperm injection; Polycystic ovary syndrome

\section{Introduction}

The treatment strategy for infertile women with polycystic ovary syndrome (PCOS) is ovulation induction with or without intrauterine insemination, followed by assisted reproduction as a further step. In vitro maturation (IVM) has emerged as an alternative treatment option for PCOS patients instead of in vitro fertilization (IVF). IVM offers several advantages over standard IVF; in particular, it eliminates the

Received: September 1, 2019 · Revised: October 15, 2019.

Accepted: October 18, 2019

Corresponding author: Pallop Pongsuthirak

Department of Obstetrics and Gynecology, Buddhachinaraj Hospital Medical

School, Phitsanulok 65000, Thailand

Tel: +66-8475-0081 E-mail: palloppong@yahoo.co.th

*This study was supported by the Hospital Endowment Fund for Medical Research.

This is an Open Access article distributed under the terms of the Creative Commons Attribution Non-Commercial License (http://creativecommons.org/licenses/by-nc/4.0/) which permits unrestricted non-commercial use, distribution, and reproduction in any medium, provided the original work is properly cited. risk of ovarian hyperstimulation syndrome, thereby lowering the cost of treatment and reducing side effects. Moreover, most children conceived following IVM treatment have shown healthy growth and development after a successful birth [1]. Many factors appear to be involved in the outcomes of IVM, including culture conditions, the cause of infertility, the source of the oocyte, and the insemination method [2]. Most oocytes cultured in vitro reach maturity within 2448 hours $[3,4]$. A longer interval may cause zona pellucida hardening, which impedes the normal fertilization process [5]; in such cases, intracytoplasmic sperm injection (ICSI) is generally advocated for assisted fertilization, rather than routine insemination [2]. At present, few studies have investigated this debatable issue, making it a challenge awaiting clarification. The purpose of this study was to compare the effects of conventional insemination and ICSI on the fertilization, developmental competence, implantation potential, and clinical pregnancy rate of embryos derived from in vitro matured oocytes of patients with PCOS. 


\section{Methods}

A prospective study was carried out at Buddhachinaraj Hospital Medical School between January 2019 to July 2019. Thirty-eight women undergoing IVM treatment were invited to participate in the study and provided written informed consent. The inclusion criteria were women $20-35$ years old who were diagnosed with PCOS by the Rotterdam criteria, had not conceived with conventional infertility treatment, and had partners with normal semen parameters. Sibling oocytes were randomly allocated into two groups: IVM with conventional insemination (IVM/IVF) and IVM with ICSI (IVM/ICSI). Embryo transfers were selected randomly between the two groups. The Research Ethics Committee approved this study (No. 34/2562) in conjunction with the Thai Clinical Trial Registry (TCTR20190817006).

\section{Treatment protocol}

In amenorrheic patients, $10 \mathrm{mg}$ of Provera was prescribed for 5 days before starting the cycle. Hormonal profiles were assessed on day 2 of the cycle. If the levels were at baseline, treatment was instituted. An ultrasound scan was carried out on days 3-4 of the cycle to exclude ovarian cysts and as a baseline record of the antral follicle count in each ovary. A dose of 100-150 IU of recombinant folliclestimulating hormone was injected subcutaneously for 3-5 days. On cycle day 6-8, an ultrasound scan was performed daily until the diameter of the follicles reached $10-12 \mathrm{~mm}$, and oocyte retrieval was then scheduled 1-2 days later. Endometrial thickness was also monitored at the same time.

\section{Oocyte retrieval, maturation, and culture}

Oocyte retrieval was performed transvaginally using a single-lumen 20-gauge aspiration needle (Cook, Sydney, Australia) connected to a vacuum pump (80-100 $\mathrm{mmHg}$ ) under ultrasound guidance. The aspirated fluid was rinsed into a sterile tissue culture dish, and examined under a dissecting microscope. The oocytes were washed with oocyte washing medium, then transferred to an oocyte maturation medium (Sage Media; Cooper Surgical, Trumbull, CT, USA). The immature oocytes were cultured in groups of up to 3 in $25-\mu \mathrm{L}$ droplets of maturation medium supplemented with $75 \mathrm{IU} / \mathrm{L}$ of human menopausal gonadotropin (IVF-M, LG Life Sciences, Iksan, Korea) under paraffin oil at $37^{\circ} \mathrm{C}$ with $6 \% \mathrm{CO}_{2}$ in humidified air for 24 hours.

\section{Sperm preparation and insemination}

Semen samples were collected by masturbation on the day following oocyte retrieval after 3-5 days of sexual abstinence. Sperm concentration, motility, and morphology were evaluated under light microscopy based on the World Health Organization criteria (fifth edition, 2010) [6]. Sperm preparation was performed using gradient centrifugation. The sperm pellet was then placed at the bottom of the fresh insemination medium and incubated at $37^{\circ} \mathrm{C}$ under $6 \% \mathrm{CO}_{2}$ to facilitate the swim-up technique. Active motile spermatozoa were harvested for insemination.

After 24 hours of culture, the oocytes in the IVM/IVF group were inseminated with 15,000 spermatozoa, while the oocytes in the IVM/ ICSI group were assessed for maturity by brief exposure to 80 IU hyaluronidase in a flushing medium and denuded of cumulus cells. The mature oocytes with first polar bodies were selected for insemination by ICSI within 2 hours.

\section{Fertilization assessment and embryo culture}

Eighteen hours after insemination, fertilization was determined by the presence of two pronuclei, and embryos were then cultured for 72 hours in $20-\mu \mathrm{L}$ drops of embryo maintenance medium in a tissue culture dish covered with paraffin oil. The embryos were then transferred into a blastocyst medium (Cook), and cultured for another 48 hours. On day 5, embryo morphology was classified at the blastocyst stage. Grade 1 blastocysts had good cellular development of both the inner cell mass (ICM) and trophectoderm (TE), grade 2 blastocysts had average development of the ICM and TE, and grade 3 had poor cellular development. Only grade 1 and 2 blastocysts were considered to be high-quality.

\section{Endometrial preparation}

Estrogen replacement therapy was prescribed to prepare the endometrium depending on its thickness [7]. If the endometrial thickness was $\geq 6 \mathrm{~mm} 2$ days before oocyte collection, the patient was orally administered 2 mg of estradiol valerate (Progynova; Schering, Berlin, Germany) three times a day. If the endometrial thickness was $<6 \mathrm{~mm}$, the estrogen dose was $3 \mathrm{mg}$ three times per day. The patients were started on twice-daily $90-\mathrm{mg}$ progesterone vaginal suppositories (Crinone; Merck-Serono, Darmstadt, Germany) on the day after oocyte retrieval and continued until the day of the pregnancy test. If the serum beta-human chorionic gonadotropin ( $\beta$-hCG) test was positive, hormonal replacement was continued until 10 weeks of pregnancy, with decreasing dosages over the last 2 weeks.

\section{Embryo transfer and pregnancy assessment}

A single blastocyst (with the highest grade) was blindly selected for transfer between the two groups under ultrasound guidance if the patient's endometrial thickness was $\geq 8 \mathrm{~mm}$. The first randomization was applied to the method of insemination, and then a blastocyst was randomly selected from the appropriate group. If the endometrial thickness was $<8 \mathrm{~mm}$, the embryos were cryopreserved for the next cycle. Pregnancy was established by a positive $\beta$-hCG test at 10 days after embryo transfer, and then patients underwent serial 
$\beta$-hCG monitoring to check for rising titers. Implantation was documented later by the appearance of the gestational sac in the uterus. Implantation and clinical pregnancy were determined by the presence of a gestational sac and fetal heartbeat, respectively, at 5 weeks after embryo transfer on transvaginal ultrasonography. Surplus highquality embryos were vitrified for future treatment.

\section{Statistical analysis}

All analyses were performed using SPSS ver. 13.0 (SPSS Inc., Chicago, IL, USA). Proportional data were compared using the chi-square test and Fisher exact test. A $p$-value of $<0.05$ was considered to indicate statistical significance. Our sample size calculation showed that at least 379 oocytes in each arm would be required to demonstrate a $10 \%$ difference in maturation rates between oocytes in the two groups, given a type I error of 5\% (two-tailed) and a type II error of $20 \%$.

\section{Results}

In total, 828 immature oocytes were collected from 42 cycles and assigned for insemination by IVF (416 oocytes) or ICSI (412 oocytes). Patients' average age was $30.4 \pm 3.7$ years, and their average body mass index was $25.5 \pm 2.6 \mathrm{~kg} / \mathrm{m}^{2}$ (Table 1). The average number of immature oocytes recovered was 20.3 \pm 5.5 . In IVF insemination, the maturation rate was $78.1 \%$ when fertilization was determined. The normal fertilization rate was $59.4 \%$ and polyspermy occurrence was $5.3 \%$. Remarkably, there were two cycles (4.8\%) in which fertilization

Table 1. Characteristics of the patients

\begin{tabular}{lc}
\hline Characteristics & Value \\
\hline Patient & 38 \\
Cycle & 42 \\
Age $(\mathrm{yr})$ & $30.4 \pm 3.7$ \\
Body mass index $\left(\mathrm{kg} / \mathrm{m}^{2}\right)$ & $25.5 \pm 2.6$ \\
\hline
\end{tabular}

Values are presented as number or mean \pm standard deviation.

Table 2. Outcomes of oocyte and embryo development using two insemination methods

\begin{tabular}{lccc}
\hline Variable & IVM/IVF & IVM/ICSI & $p$-value \\
\hline Immature oocyte & 416 & 412 & - \\
Mature oocyte $^{\text {b) }}$ & $325(78.1)$ & $299(72.6)$ & 0.076 \\
Normal fertilization & $193(59.4)$ & $200(66.9)$ & 0.063 \\
Blastocyst & $117(60.6)$ & $107(53.5)$ & 0.186 \\
High-quality blastocyst & $79(40.9)$ & $93(46.5)$ & 0.314 \\
\hline
\end{tabular}

Values are presented as number (\%).

IVM, in vitro maturation; IVF, in vitro fertilization; ICSI, intracytoplasmic sperm injection.

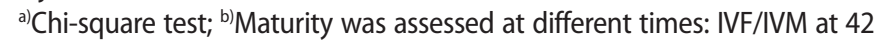
hours and IVM/ICSI at 24 hours after oocyte collection. totally failed. The blastocyst formation rate was $60.6 \%$. Meanwhile, in the ICSI group, the maturation rate prior to insemination was $72.6 \%$ and some oocytes degenerated after ICSI (2.1\%). The fertilization rate was $66.9 \%$ and the blastocyst formation rate was $53.5 \%$ (Table 2). The implantation and clinical pregnancy rates were comparable between the two insemination methods (Table 3).

\section{Discussion}

A few previous studies have compared the method of insemination in IVM. Hwang et al. [3], in an experimental study using a TCM medium for oocytes collected from cesarean sections with 48 hours of maturation, reported a fertilization rate of $84.1 \%$ by ICSI of cumulusenclosed oocytes, which was significantly higher than the rate achieved using conventional insemination (56.5\%); in cumulus-denuded oocytes, the fertilization rate was $84.5 \%$ by ICSI and $39.5 \%$ by IVF, respectively. The researchers suggested that ICSI is the preferential method of fertilization. Soderstrom-Anttila et al. [8], in a retrospective study using TCM-199 and Medcult medium with 30 hours of culture also found that the fertilization rate was higher with ICSI than with IVF (78.4\% vs. $43.8 \%$, respectively) among PCOS patients. In our study, we found a contradictory result using Sage IVM medium as the maturation agent of oocytes for 24 hours. Our results showed comparable maturation and fertilization rates to those reported by Wall et al. [4] using G-2Plus medium (Vitrolife, Gothenburg, Sweden) with 24 hours of culture before insemination, who demonstrated a $71.8 \%-79.2 \%$ maturation rate and an insignificant difference in the fertilization rate between ICSI (67.9\%) and IVF (59.6\%) [4]. These discrepancies can be partially explained by differences in patient selection, culture media, and the duration of IVM. Our previous study found no significant difference between Sage IVM medium and Cook blastocyst media used for IVM [9], and the fact that G-2Plus medium is a blastocyst medium may have given rise to the similarity between our results and their findings. However, future research into ways of optimizing the fertilization rate remains necessary.

Table 3. Embryonic outcomes according to the insemination method

\begin{tabular}{lccc}
\hline Variable & IVM/IVF & IVM/ICSI & $p$-value \\
\hline High-quality blastocyst & 76 & 93 & $\mathrm{NS}$ \\
Vitrified blastocyst & 55 & 72 & $\mathrm{NS}$ \\
Embryos transferred & 21 & 21 & - \\
Fresh cycle & 18 & 19 & $\mathrm{NS}$ \\
Cryopreserved cycle & 3 & 2 & $\mathrm{NS}^{\text {b) }}$ \\
Implantation & $11(52.2)$ & $10(47.6)$ & $\mathrm{NS}$ \\
Clinical pregnancy & $9(42.9)$ & $9(42.9)$ & $\mathrm{NS}$ \\
\hline
\end{tabular}

IVM, in vitro maturation; IVF, in vitro fertilization; ICSI, intracytoplasmic sperm injection; NS, not significant.

${ }^{\text {a) }}$ Chi-square test; ${ }^{\text {b) }}$ Fisher exact test. 
In fact, a longer period of maturation not only produces more mature oocytes, but also potentiates more zona hardening, which may obscure normal fertilization [5]. Zhang et al. [10] reported more chromosomal abnormalities derived from in vitro matured oocytes at 48 hours than at 24 hours (100\% vs. $67 \%$, respectively), which may have caused poor outcomes in the oocytes with longer maturation times. In this study, we inseminated the cumulus-enclosed oocytes after a 24-hour period of maturation, which was shorter than the maturation periods used in previous studies [3,8], and obtained a satisfactory fertilization rate with IVF insemination. Further studies are required to elucidate the impact of different durations of IVM at the time of IVF insemination. Nevertheless, total fertilization failure (TFF) may occur when utilizing IVF insemination because the oocytes derived from PCOS patients might be prone to fertilization failure, both due to the poor quality of the oocytes resulting from a long exposure to an abnormal endocrine milieu [11] and intrinsic aberrations during folliculogenesis [12]. Accordingly, we found a $4.8 \%$ rate of TFF in the IVF group, but no cases of TFF in the ICSI group. However, the rate of TFF was not significantly different from that of oocyte maturation in vivo, which has been found to be $5 \%-10 \%$ [13]. Interestingly, the sibling oocytes exhibiting TFF during IVF insemination displayed normal fertilization by ICSI because ICSI skips over the elaborate cascades of interactions between sperm and cumulus-oocyte complexes and deposits the sperm directly inside the ooplasm. In this context, ICSI may therefore offer more potential for the oocyte to fertilize and develop to the embryonic stage $[13,14]$, even though ICSI itself cannot preclude TFF.

During IVF insemination, cumulus cells may play an important role in fertilization [3,15]. Hwang et al. [3] reported that cumulus-enclosed oocytes had a higher fertilization rate than cumulus-denuded oocytes (56.5\% vs. 39.5\%) when oocytes were fertilized by IVF. Therefore, IVF insemination must be performed without the denudation of cumulus cells to evaluate the maturation of the oocytes. As a result, it might not be accurate to directly compare the maturation rate of the oocytes at the time of ICSI (24 hours) and fertilization determination in IVF insemination (36 hours) because maturation was assessed at different times.

Currently, embryos derived from IVM can continue being cultured to the blastocyst stage and embryo transfer may be carried out at this point with promising results $[2,7]$. Blastocyst development is a reliable way to predict the success rates of implantation and ongoing pregnancy [8]. In our study, no significant difference was found in the blastocyst formation, implantation, and clinical pregnancy rates between IVF and ICSI (Table 3), similarly to a previous report [5]. Nevertheless, the implantation and clinical pregnancy rate may also depend on endometrial preparation. In earlier studies, a low success rate for IVM was reported because of discrepancies between endo- metrial thickness and embryonic development [2,7]. We started hormonal replacement using a dose based on the endometrial thickness 2 days prior to oocyte retrieval, with the goal of ensuring an adequate thickness before embryo transfer, as recommended by Junk and Yeap [7]. To increase the chance of pregnancy, embryos can be vitrified for the next freeze-thaw cycle if the endometrium does not attain a suitable thickness.

In this study, we obtained a comparable yield of in vitro matured oocytes derived from PCOS patients in terms of the fertilization, blastocyst formation, and implantation rates between IVF and ICSI insemination. These findings offer valuable insights for choosing an assisted reproductive treatment for women with PCOS, as IVM has promising outcomes and is less invasive and less costly.

\section{Conflict of interest}

No potential conflict of interest relevant to this article was reported.

\section{Acknowledgments}

The author thanks Mr. Robert Moore, a professional English reviewer, for his kind help in editing this manuscript.

\section{ORCID}

Pallop Pongsuthirak https://orcid.org/0000-0002-6068-2256

\section{References}

1. Fadini R, Mignini Renzini M, Guarnieri T, Dal Canto M, De Ponti E, Sutcliffe A, et al. Comparison of the obstetric and perinatal outcomes of children conceived from in vitro or in vivo matured oocytes in in vitro maturation treatments with births from conventional ICSI cycles. Hum Reprod 2012;27:3601-8.

2. Julania $S$, Walls $M L$, Hart R. The place of in vitro maturation in PCO/PCOS. Int J Endocrinol 2018;2018:5750298.

3. Hwang JL, Lin YH, Tsai YL. In vitro maturation and fertilization of immature oocytes: a comparative study of fertilization techniques. J Assist Reprod Genet 2000;17:39-43.

4. Walls M, Junk S, Ryan JP, Hart R. IVF versus ICSI for the fertilization of in-vitro matured human oocytes. Reprod Biomed Online 2012;25:603-7.

5. Dietzel E, Wessling J, Floehr J, Schafer C, Ensslen S, Denecke B, et al. Fetuin- $B$, a liver-derived plasma protein is essential for fertilization. Dev Cell 2013;25:106-12.

6. World Health Organization. WHO laboratory manual for the examination and processing of human semen. 5th ed. Geneva: 
WHO Press; 2010.

7. Junk SM, Yeap D. Improved implantation and ongoing pregnancy rates after single-embryo transfer with an optimized protocol for in vitro oocyte maturation in women with polycystic ovaries and polycystic ovary syndrome. Fertil Steril 2012;98:888-92.

8. Soderstrom-Anttila V, Makinen S, Tuuri T, Suikkari AM. Favourable pregnancy results with insemination of in vitro matured oocytes from unstimulated patients. Hum Reprod 2005;20:153440.

9. Pongsuthirak P, Songveeratham S, Vutyavanich T. Comparison of blastocyst and Sage media for in vitro maturation of human immature oocytes. Reprod Sci 2015;22:343-6.

10. Zhang XY, Ata B, Son WY, Buckett W, Tan SL, Ao A. The chromosome constitution of IVM embryos generated from oocytes at different maturation interval. Fertil Steril 2010;94;S142-3.

11. Kodama H, Fukuda J, Karube H, Matsui T, Shimizu Y, Tanaka T.
High incidence of embryo transfer cancellations in patients with polycystic ovarian syndrome. Hum Reprod 1995;10:1962-7.

12. Teixeira Filho FL, Baracat EC, Lee TH, Suh CS, Matsui M, Chang RJ, et al. Aberrant expression of growth differentiation factor-9 in oocytes of women with polycystic ovary syndrome. J Clin Endocrinol Metab 2002;87:1337-44.

13. Kahyaoglu I, Demir B, Turkkanı A, Cinar O, Dilbaz S, Dilbaz B, et al. Total fertilization failure: is it the end of the story? J Assist Reprod Genet 2014;31:1155-60.

14. Simopoulou M, Giannelou P, Bakas P, Gkoles L, Kalampokas T, Pantos K, et al. Making ICSI safer and more effective: a review of the human oocyte and ICSI practice. In Vivo 2016;30:387-400.

15. Hyun CS, Cha JH, Son WY, Yoon SH, Kim KA, Lim JH. Optimal ICSI timing after the first polar body extrusion in in vitro matured human oocytes. Hum Reprod 2007;22:1991-5. 